\title{
Reduction in Sporadic Norovirus Infections Following the Start of the COVID-19 Pandemic, 2019-2020, Philadelphia
}

\author{
Irving Nachamkin (D) - Melissa Richard-Greenblatt • Mei Yu • \\ Hong Bui
}

Received: April 16, 2021 / Accepted: May 27, 2021 / Published online: June 13, 2021

(C) The Author(s) 2021

\section{ABSTRACT}

Introduction: Norovirus infections are common in the USA and worldwide. Detection of norovirus in fecal samples is now common in routine tests for enteric pathogens using molecular methods. We observed a change in positivity rates for norovirus after the beginning of the coronavirus disease 2019 (COVID-19) pandemic in our laboratory and performed a more detailed analysis of testing results.

Methods: We reviewed the positivity rates for detection of common enteric pathogens from stool samples submitted to an academic medical center laboratory pre (2016-2019) and post the start of the COVID-19 pandemic (2020).

Results: In contrast to other enteric pathogens, norovirus positivity rates dropped dramatically from a yearly average of $3.9 \%$ in 2016-2019 to $0.76 \%$ from March 2020 through the end of 2020 .

I. Nachamkin $(\bowtie) \cdot$ M. Richard-Greenblatt Division of Laboratory Medicine, Department of Pathology and Laboratory Medicine, Perelman School of Medicine, University of Pennsylvania, 4th Floor Gates Pavilion, 3400 Spruce Street, Philadelphia, PA 19104-4283, USA

e-mail: nachamki@pennmedicine.upenn.edu

I. Nachamkin · M. Richard-Greenblatt - M. Yu . H. Bui

Clinical Microbiology Laboratory, Hospital of the University of Pennsylvania, Philadelphia, PA 191044283, USA
Conclusion: A sustained reduction in norovirus positivity rates was temporally associated with COVID-19 mitigation processes in the Philadelphia area, while positivity rates for other common enteric pathogens were only intermittently reduced.

Keywords: COVID-19; Diagnostic microbiology; Enteric infections; Gastrointestinal infections; Norovirus

\section{Key Summary Points}

We analyzed the positivity rates for norovirus and other enteric pathogens from 2016 to 2020.

We observed that norovirus rates were dramatically reduced and sustained in 2020 coinciding with the onset of the COVID-19 pandemic and mitigation factors to prevent COVID-19.

Initial reductions in positive rates for other enteric pathogens were observed for several months at the beginning of the COVID-19 pandemic, but returned to historical rates.

The study provides some insight into the mechanisms for norovirus infection reduction associated with interventions to prevent COVID-19 infections during the past year. 


\section{DIGITAL FEATURES}

This article is published with digital features, including a summary slide, to facilitate understanding of the article. To view digital features for this article go to https://doi.org/10.6084/ m9.figshare.14680902.

\section{INTRODUCTION}

Norovirus infections are one of the most common causes of viral gastrointestinal infections in the USA. While most infections are sporadic community-acquired infections, outbreaks of norovirus in the USA are frequent as reported through the Centers for Disease Control and Prevention (CDC) National Outbreak Reporting System (NORS) [1]. Norovirus is primarily transmitted via fecal-oral route, and commonly contaminates inanimate surfaces associated with ill individuals. Surface decontamination and handwashing are mitigation procedures to help reduce norovirus [2].

In the past 5 years, clinical microbiology laboratories have been able to routinely detect norovirus in fecal samples using a number of commercially available multiplex nucleic acid amplification test platforms [3]. Previously, testing was only available through reference laboratories or public health laboratories. Norovirus is the most common enteric pathogen detected in our laboratory with a 3-5\% average annual positivity rate. Following the initial months of the coronavirus disease 2019 (COVID-19) pandemic, we started to observe a reduction in positivity rates for norovirus as well as other enteric pathogens. However, norovirus positivity rates have remained at a very low level through the end of 2020 whereas others have returned to pre-pandemic levels. Here we report the details of our pre- and postpandemic norovirus testing results.

\section{METHODS}

The analysis reported here was performed using monthly data for detecting common bacterial and viral enteric pathogens in the Clinical
Microbiology Laboratory at the Hospital of the University of Pennsylvania (HUP, site 1), a major academic medical center serving the Philadelphia region. The laboratory performs all microbiological testing (enteric and non-enteric) for two hospitals, HUP, an 839-bed hospital, and Penn Presbyterian Medical Center (site 2), a 375-bed hospital near the HUP campus; both hospitals are part of the University of Pennsylvania Health System (UPHS), a six-hospital system in the PA, NJ region. One other UPHS hospital, Pennsylvania Hospital (site 3), also located in the city of Philadelphia, a 525-bed hospital, regularly sends fecal samples to the HUP laboratory for enteric pathogen testing. Outpatient UPHS clinics are either mapped to each hospital location (site 1, site 2, site 3 ) or have their own separate location. Other fecal samples for enteric pathogen testing sent to the HUP laboratory come from a variety of UPHS-owned outpatient facilities across the Philadelphia region. In 2019 and 2020, 96.7\% and $94 \%$ of fecal samples submitted for norovirus testing were from patients either seen in outpatient clinics or from inpatients tested within 3 days after admission, respectively. All samples for enteric pathogen testing sent to the HUP clinical microbiology laboratory were tested using the Verigene ${ }^{\circledR}$ Enteric Pathogens Test (Luminex Corp, Austin, TX). This molecular multiplex PCR panel tests for nine different targets, Campylobacter, Salmonella, Shigella, Vibrio, Yersinia, Shiga toxin 1 (Stx1), Shiga toxin 2 (Stx2), norovirus, and rotavirus with an approximate $2 \mathrm{~h}$ running time to results.

For statistical analysis, the difference in proportion of requests or proportion of tests positive for an enteropathogen between 2019 and 2020 were computed using the chi-square test of homogeneity (test of two proportions) using MedCalc Version 19.8. Statistical significance in proportion of requests between 2019 and 2020 was calculated using proportion of requests (\%) per site and sum of tests ordered for the respective year. In contrast, proportion of positive cases (\%) and sum of tests ordered per site for each year were used to determine significance. $P<0.05$ was considered significant for all comparisons. 
This analysis reported here was exempt and not considered to meet criteria for human research requiring IRB review by the University of Pennsylvania Institution Review Board.

\section{RESULTS}

We analyzed the number of tests ordered and proportion of test requests from 11 different UPHS locations during 2019 (pre-COVID) and 2020 (post-COVID) submitting samples to the laboratory for testing. The total number of samples tested dropped approximately 25\% from $2019(n=3842)$ to $2020(n=2896)$. The proportion of test orders from sites with 25 or more test orders $(n=7)$ from 2019 to 2020 were essentially unchanged $(P \geq 0.05)$, except for a slight increase in the proportion of tests ordered from site 1 locations (58.7 vs $62.4 \%, P<0.0001$ ) and site 7 locations $(0.6 \%$ vs $1.1 \%$, $P<0.05$ ) (Table 1).

The yearly positivity rates for norovirus, Campylobacter, Salmonella, and Shigella from 2016 through 2020 are shown in Fig. 1. From 2016 through 2020, the number of tests performed for enteric pathogens was 3053, 3578, 3815, 3842, and 2896 respectively. Campylobacter yearly positivity rates varied between $1.8 \%$ and $3.0 \%$ pre-COVID-19, dropped to $1.1 \%$ or less in March-May 2020, then returned to higher rates the remainder of 2020 (Fig. 2). For Salmonella, average yearly pre-COVID-19 positive rates ranged from $1.3 \%$ to $1.5 \%$, dropped in March-May 2020, then returned to historical levels. For Shigella, historical yearly positive rates were $1.0-1.2 \%$ pre-COVID-19. Between March and May 2020, two of the 3 months showed rates less than $1.0 \%$, then returned to historical levels. For norovirus, pre-COVID-19 yearly rates ranged from $3.5 \%$ to $4.0 \%$ (mean $3.9 \%$ ). From 2019, the proportion of norovirus cases dropped significantly to $1.4 \%$ overall in 2020 from March to December 2020 $(P<0.0001)$. From March through the end of December 2020, positive rates were approximately $1 \%$ or less, with 5 months at less than $0.5 \%$ positive and a rate of $0.4 \%$ in December 2020. Norovirus positivity rates dropped across all locations submitting samples for testing. For
Table 1 Proportion of site-specific requests for enteric test ordering across University of Pennsylvania Health System facilities (2019-2020)

\begin{tabular}{lll}
\hline Site & \multicolumn{2}{l}{ Proportion of requests $(\%)^{\mathbf{a}, \mathbf{b}}$} \\
\cline { 2 - 3 } & $\mathbf{2 0 1 9}$ & $\mathbf{2 0 2 0}$ \\
\hline 1 & 58.7 & $62.4^{* *}$ \\
2 & 16.9 & 16.8 \\
3 & 12.3 & 11.2 \\
4 & 7.8 & $5.2^{*}$ \\
5 & 2.0 & 1.5 \\
6 & 1.3 & 1.1 \\
7 & 0.6 & $1.1^{* * *}$ \\
8 & 0.2 & 0.4 \\
9 & 0.2 & 0.07 \\
10 & 0.05 & 0.0 \\
11 & 0.03 & 0.0 \\
\hline
\end{tabular}

a Proportions of requests were calculated using the tests ordered by site and total tests ordered across all University of Pennsylvania Health System facilities each year. Total tests ordered in $2019(n=3842)$ and $2020(n=2896)$

b Differences in proportion of requests between 2019 and 2020 were calculated using the proportion of requests (\%) at each site and sum of tests ordered annually across all sites. $P$ values were computed using the chi-square test of homogeneity (test of two proportions) MedCalc Version 19.8

${ }^{*} P<0.01,{ }^{* *} P<0.0001,{ }^{* * *} P<0.05$

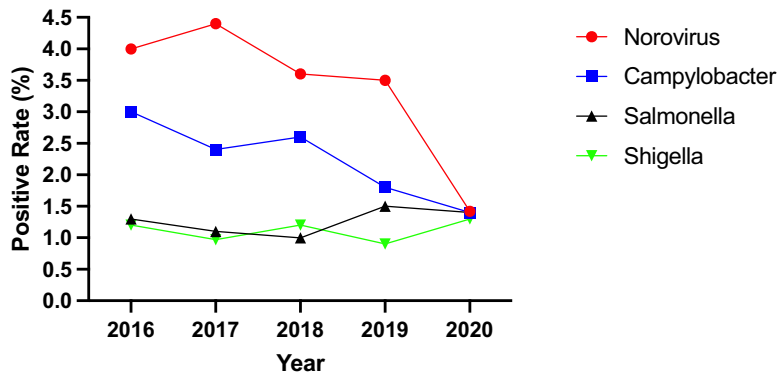

Fig. 1 Norovirus, Campylobacter, Salmonella, and Shigella yearly positivity rates among fecal samples submitted to the HUP Clinical Microbiology Laboratory, 2016-2020 
Norovirus

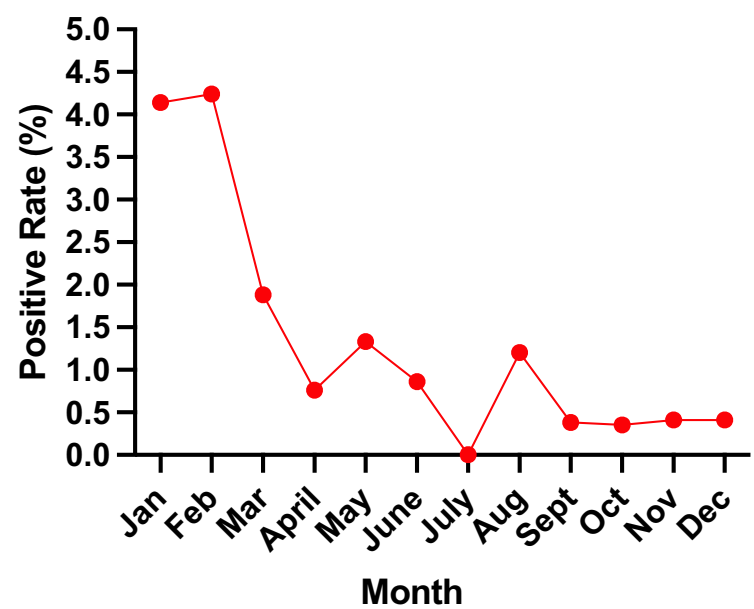

Salmonella

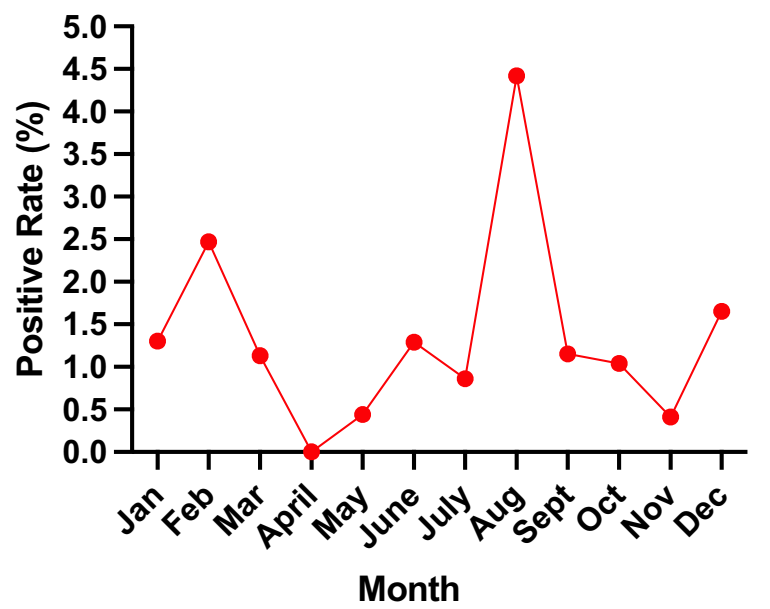

\section{Campylobacter}

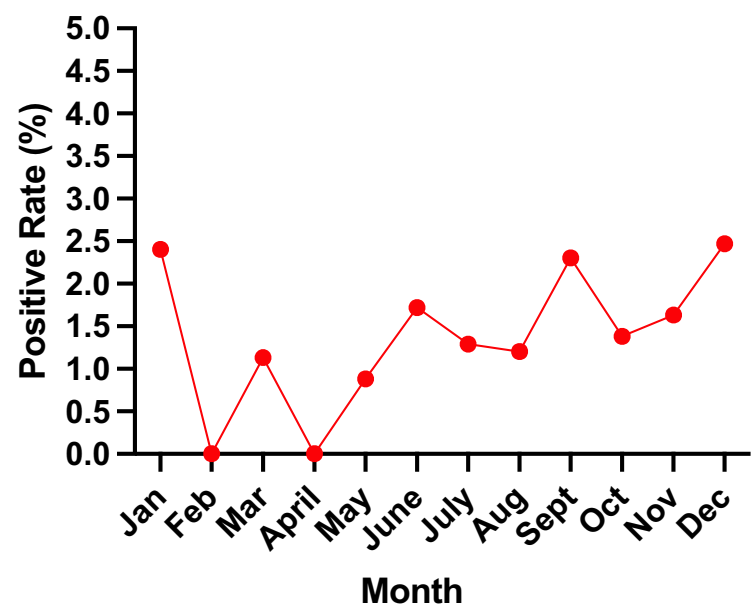

Shigella

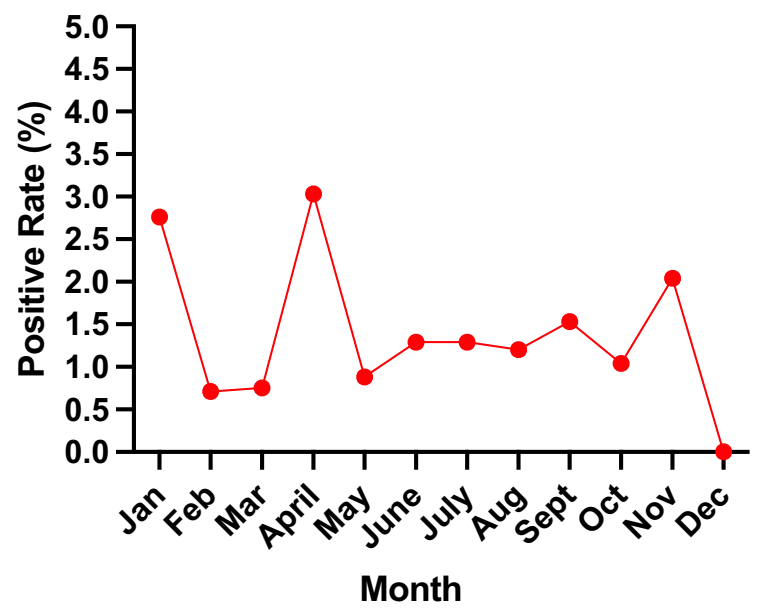

Fig. 2 Monthly positivity rates for norovirus, Campylobacter, Salmonella, and Shigella among fecal samples submitted to the HUP Clinical Microbiology Laboratory, 2020

other enteric pathogens included in the Verigene $^{\circledR}$ multiplex panel (Fig. 3), the only significant drop from 2019 to 2020 was with rotavirus, although the total number of positives was small $(0.8-0.2 \%, P<0.001)$. There were no significant differences year-year for Vibrio $(0.2 \%$ vs $0.1 \%)$, Yersinia $(0.3 \%$ vs $0.2 \%)$, or Shiga toxin 1 and Shiga toxin $2(0.7 \%$ vs $0.4 \%)$.

\section{DISCUSSION}

Decreased norovirus activity in 2020 has been noted across the globe, particularly in the number of recorded norovirus outbreaks. Douglas et al. recently reported sharp reductions (greater than 80\%) in cases based on data from multiple surveillance programs in England [4]. Eigner et al. reported a sharp decrease in the proportion of hospitalized patients in Germany 


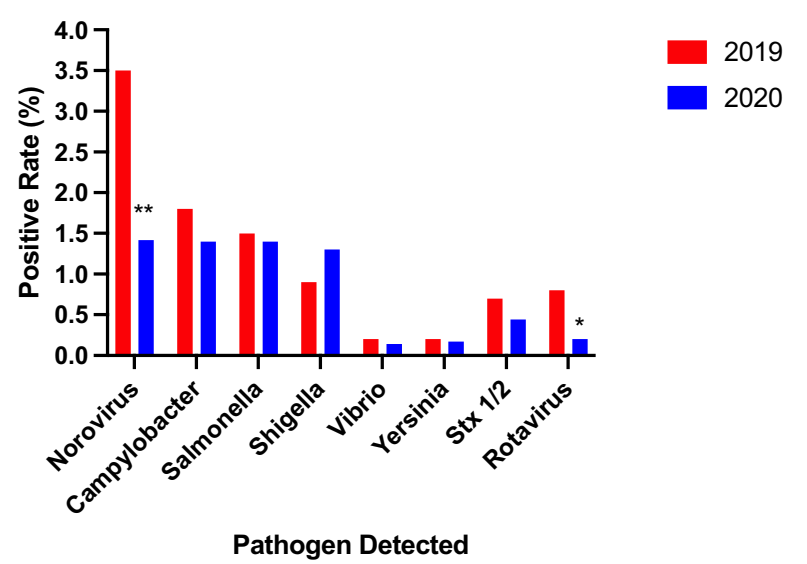

Fig. 3 Positivity rates for bacterial and viral enteric pathogens in the Verigene ${ }^{\circledR}$ multiplex gastrointestinal pathogen panel among fecal samples submitted for testing in the Clinical Microbiology Laboratory, HUP, 2019-2020. ${ }^{*} P<0.001,{ }^{* *} P<0.0001$

positive for norovirus (less than 1.0\%) [5]. Similarly, the number of norovirus associated outbreaks dramatically declined in Australia after the COVID-19 pandemic started in 2020 compared with previous years [6]. Outbreaks of norovirus in the USA have also been curtailed post COVID-19 as reported by Kraay et al. in a nine-state surveillance system [7] as well as an analysis of pre- and post-COVID-19 outbreaks using data from the CDC NORS [8]. We are unaware of published reports of sporadic norovirus infections in the USA since the beginning of the COVID-19 pandemic.

COVID-19 mitigation orders have been in effect in Pennsylvania since March 6, 2020 when the PA Governor declared a disaster emergency and the declaration expired on May 31, 2021. The mandated masking order was issued in July 2020 and will be lifted when 70\% of Pennsylvania adults are fully vaccinated; the order is still in place. In Philadelphia, relaxed stay-at-home restrictions went into effect on May 7, 2021 [9].

COVID-19 health safety measures including stay-at-home orders, social distancing, and hand hygiene were temporally associated with a decline in norovirus positivity rates. In addition, COVID-19 mitigation efforts were also associated with brief declines in positivity rates for other enteric pathogens. However, our study was observational. Therefore, we cannot definitively state that these measures either alone, or in combination with other unknown activities, caused the decrease in positivity rates for the different pathogens. Handwashing, stayat-home orders, and sharing food may have been activities to help reduce foodborne illnesses, but are not the only activities involved with preventing common foodborne pathogen infections. Safe food practices such as thorough cooking and prevention of cross-contamination are other activities that may not have been impacted during the past year. Norovirus has a low infectious dose; its main mechanism of transmission is fecal-oral and is more likely to be impacted by COVID-19 healthy safety measure than other enteropathogens [10]. However, these differences do not fully explain why we observed a return to historical rates of bacterial enteric pathogens after only a few months following the institution of national stay-at-home mandates, attention to mask wearing, and hand hygiene.

\section{CONCLUSIONS}

Our observations on sustained reduction in the positivity rate for norovirus and intermittent reductions for other enteric pathogens were temporally associated with COVID-19 mitigation efforts, but not proven by the nature of our study design. Further research on factors leading to the reduction of bacterial enteric infections noted above may provide clues to our observations.

\section{ACKNOWLEDGEMENTS}

Funding. No funding or sponsorship was received for this study or publication of this article.

Authorship. All named authors meet the International Committee of Medical Journal Editors criteria for authorship for this article, take responsibility for the integrity of the work 
as a whole, and give their approval for this version to be published.

Authorship Contribution. Drs Nachamkin and Richard-Greenblatt - study and manuscript preparation, Ms Yu and Ms Bui worked on data analytics.

Disclosures. None of the authors have any financial or other conflicts of interest to disclose.

Compliance with Ethics Guidelines. This analysis reported here was exempt and not considered to meet criteria for human research requiring IRB review by the University of Pennsylvania Institution Review Board.

Data Availability. The datasets analyzed during the current study are available from the corresponding author on reasonable request.

Author Bio. Dr Nachamkin is currently Emeritus Professor of Pathology and Laboratory Medicine and the immediate past director of the division of laboratory medicine and associate director of the clinical microbiology laboratory.

Open Access. This article is licensed under a Creative Commons Attribution-NonCommercial 4.0 International License, which permits any non-commercial use, sharing, adaptation, distribution and reproduction in any medium or format, as long as you give appropriate credit to the original author(s) and the source, provide a link to the Creative Commons licence, and indicate if changes were made. The images or other third party material in this article are included in the article's Creative Commons licence, unless indicated otherwise in a credit line to the material. If material is not included in the article's Creative Commons licence and your intended use is not permitted by statutory regulation or exceeds the permitted use, you will need to obtain permission directly from the copyright holder. To view a copy of this licence, visit http://creativecommons.org/licenses/by$\mathrm{nc} / 4.0 /$.

\section{REFERENCES}

1. Centers for Disease Control and Prevention (CDC). National outbreak reporting system. Atlanta, Georgia: U.S. Department of Health and Human Services, CDC. https://wwwn.cdc.gov/norsdashboard/. Accessed 12 April 2020.

2. Pang $X$, Lee BE. Laboratory diagnosis of noroviruses: present and future. Clin Lab Med. 2015;35:345-62. https://doi.org/10.1016/j.cll.2015. 02.008 .

3. Ramanan P, Bryson AL, Binnicker MJ, Pritt BS, Patel R. Syndromic panel-based testing in clinical microbiology. Clin Microbiol Rev. 2018;31:e00024e117. https://doi.org/10.1128/CMR.00024-17.

4. Douglas A, Sandmann FG, Allen DJ, Celma CC, Beard S, Larkin L. Impact of COVID-19 on national surveillance of norovirus in England and potential risk of increased disease activity in 2021. J Hosp Infect. 2021. https://doi.org/10.1016/j.jhin.2021. 03.006 .

5. Eigner U, Verstraeten T, Weil J. Decrease in norovirus infections in Germany following COVID- 19 containment measures. J Infect. 2021. https://doi. org/10.1016/j.jinf.2021.02.012.

6. Brguggink LD, Garcia-Clapes A, Tran T, Druce JD, Thorley BR. Decreased incidence of enterovirus and norovirus infections during the COVID-19 pandemic, Victoria, Australia, 2020. Commun Dis Intell. 2021. https://doi.org/10.33321/cdi.2021.45. 5.

7. Kraay ANM, Han P, Kambhampati AK, Wikswo ME, Mirza SA, Lopman BA. Impact of nonpharmaceutical interventions for severe acute respiratory syndrome coronavirus 2 on norovirus outbreaks: an analysis of outbreaks reported by 9 US state. J Infect Dis. 2021. https://doi.org/10.1093/infdis/jiab093.

8. Lennon RP, Griffin C, Miler EL, Dong H, Rabago D, Zgierska AE. Norovirus infections drop 49\% in the United States with strict COVID-19 public health interventions. Acta Med Acad. 2020;49(3):278-80. https://doi.org/10.5644/ama2006-124.317.

9. City of Philadelphia. Coronavirus disease 2019 (COVID-19). 2021. https://www.phila.gov/ programs/coronavirus-disease-2019-covid-19/. Accessed 20 May 2021.

10. Gaythorpe KAM, Trotter CL, Lopman B, Steele M, Conlan AJK. Norovirus transmission dynamics: a modelling review. Epidemiol Infect. 2018;146(2): 147-58. https://doi.org/10.1017/ 